\title{
Concordancia entre las escalas Marshall, Ranson y APACHE II como estimadoras de la morbimortalidad en pancreatitis aguda
}

\section{Agreement between Marshall, Ranson and Apache II as estimators of morbidity and mortality in acute pancreatitis}

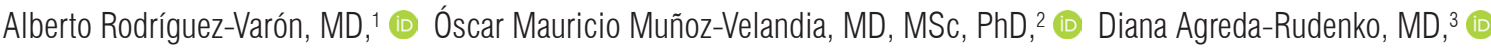 \\ Elías García-Consuegra, MD. $4^{*}$
}

\author{
GacCeso abierto \\ Citación: \\ Rodríguez-Varón A, Muñoz-Velandia OM, Agreda- \\ Rudenko D, García-Consuegra E. Concordancia \\ entre las escalas Marshall, Ranson y APACHE \\ II como estimadoras de la morbimortalidad \\ en pancreatitis aguda. Rev Colomb \\ Gastroenterol. 2020;35(3):298-303. https://doi. \\ org/10.22516/25007440.457
}

Médico internista, gastroenterólogo. Profesor titular de Gastroenterología y Medicina Interna, Departamento de Medicina Interna. Unidad de gastroenterología clínica y endoscopia digestiva, Pontificia Universidad Javeriana, Hospital Universitario San Ignacio; Bogotá, Colombia.

Médico internista y epidemiólogo clínico. Profesor de Medicina Interna, Departamento de Medicina Interna, Pontificia Universidad Javeriana, Hospital Universitario San Ignacio; Bogotá, Colombia.

3 Médica internista. Departamento de Medicina Interna, Pontificia Universidad Javeriana, Hospital Universitario San Ignacio; Bogotá, Colombia.

${ }^{4}$ Residente de Medicina Interna, Departamento de Medicina Interna, Hospital Universitario San Ignacio, Pontificia Universidad Javeriana; Bogotá, Colombia.

"Correspondencia: Elías García-Consuegra, MD Elias_gc10@hotmail.com

Fecha recibido: $\quad 26 / 08 / 19$ Fecha aceptado: 30/09/19

\section{Resumen}

Objetivo: actualmente, en Colombia están en uso diferentes escalas para estimar el riesgo de morbimortalidad en pacientes con pancreatitis, lo que genera incertidumbre a la hora de clasificar y manejar a estos pacientes. El objetivo de este estudio es analizar la concordancia entre las más usadas en una población atendida a 2670 metros sobre el nivel del mar (msnm). Materiales y métodos: se evaluaron 200 pacientes, entre 18 y 65 años, con diagnóstico de pancreatitis aguda y manejados en el Hospital Universitario San Ignacio de Bogotá (Colombia). Se estimaron tres escalas de riesgo y se clasificaron como pancreatitis con predicción de gravedad si los puntajes de APACHE II eran $\geq 8$, Marshall modificada $\geq 2$, o si tenían 3 o más criterios de Ranson positivos. Se determinó la concordancia entre los resultados usando el estadístico kappa. Resultados: según Marshall, el $45,5 \%$ de los pacientes correspondieron a pancreatitis con predicción de gravedad, mientras que de acuerdo con APACHE II y Ranson se encontró este diagnóstico en un 39,5\% y un 38,5\%, respectivamente. El coeficiente kappa mostró una concordancia débil entre APACHE II y Ranson (kappa: 0,201; intervalo de confianza [IC], 95 $\%: 0,05-0,34$ ), así como una concordancia pobre entre Ranson y Marshall (kappa: 0,18; IC, $95 \%$ : 0,04-0,32). La concordancia entre APACHE y Marshall fue moderada (kappa: 0,42; IC, $95 \%$ : 0,28-0,56). Conclusiones: existe un pobre acuerdo entre las diferentes escalas de clasificación de riesgo de pancreatitis usadas en Colombia, por lo que no pueden interpretarse como clínicamente equivalentes. Los datos de este estudio demuestran la necesidad de validar las distintas escalas en Colombia y en Latinoamérica. Además, sugieren que la escala de Marshall sobreestima el riesgo en ciudades por encima de los $2000 \mathrm{msnm}$.

\section{Palabras clave}

Pancreatitis aguda, concordancia, mortalidad, modelos de predicción multivariados.

\section{Abstract}

Introduction: Different scales to estimate the risk of morbidity and mortality in patients with pancreatitis are currently in use in Colombia, which leads to uncertainty when classifying and treating these patients. Objective: This study seeks to analyze agreement between the most used scales to estimate the risk of patient morbidity and mortality in a population treated at 2,670 meters above sea level (m.a.s.l.). Materials and methods: Two hundred patients between 18 and 65 years old, diagnosed with acute pancreatitis, were evaluated and treated at the Hospital Universitario San Ignacio, Bogotá (Colombia). Three risk scales were used for the estimations. Scores $\geq 8$ in the APACHE II system, $\geq 2$ in the Modified Marshall Score, or 3 or more positive Ranson criteria were classified as pancreatitis with severity prognostic marker. Agreement between the results was determined using the Kappa coefficient. Results: According to the Marshall score, $45.5 \%$ of the cases were pancreatitis with predicted severity, while APACHE II and Ranson yielded scores of $39.5 \%$ and $38.5 \%$, respectively. The Kappa coefficient showed weak agreement between APACHE II and Ranson (Kappa=0.201; 95\% Cl 0.05-0.34), poor agreement between Ranson and Marshall (Kappa=0.18; 95\% Cl 0.04-0.32), and moderate agreement between APACHE II and Marshall (Kappa $=0.42 ; 95 \% \mathrm{Cl} 0.28-0.56$ ). Conclusions: There is poor agreement between the pancreatitis severity scoring systems used in Colombia, so they cannot be interpreted as clinically equivalent. The data from this study demonstrate the need to validate the scales in Colombia and Latin America. They also suggest that the Marshall scale overestimates the risk in cities above 2,000 m.a.s.I.

\section{Keywords}

Acute pancreatitis; Agreement; Mortality; Multivariate prediction models. 


\section{INTRODUCCIÓN}

En la valoración inicial de los pacientes con pancreatitis aguda es muy importante la detección temprana de la disfunción o de la falla multiorgánica. Por tanto, debe considerarse que los pacientes que presentan esta patología se benefician con intervenciones diagnósticas y terapéuticas más invasivas, encaminadas a modificar el curso de la enfermedad y reducir su morbimortalidad (1).

Para identificar a los pacientes con riesgo de disfunción multiorgánica se ha creado una serie de escalas que los clasifican con base en datos clínicos y paraclínicos. Dentro de las más utilizadas se encuentran las escalas APACHE II (2), Ranson (3) y Marshall (4). Cada una de ellas tiene ventajas y desventajas. La de APACHE II, por ejemplo, es dinámica y permite estadificar el riesgo según la evolución del paciente, pero es dispendiosa de realizar y requiere la toma de múltiples paraclínicos. Entre tanto, la de Ranson ha sido utilizada durante mucho tiempo y es familiar para una gran cantidad de médicos; sin embargo, requiere de una valoración completa después de 48 h, a fin de poder ser calculada, por lo que sus resultados están disponibles de forma tardía. Y la de Marshall permite la valoración de distintos sistemas (renal, cardiovascular y respiratorio) y es fácil del calcular; no obstante, no ha sido validada en nuestro entorno.

En este contexto, dichas escalas se usan simultáneamente, lo que implica que los pacientes pueden quedar clasificados de forma diferente por cada una. Esta situación genera incertidumbre en el médico con respecto a cuál de esas estimaciones de riesgo deben usarse con miras a ofrecer el mejor manejo posible a los enfermos.

Hasta el momento, no se ha evaluado si se puede asumir que las diferentes escalas para evaluar el riesgo de morbimortalidad en pancreatitis aguda son clínicamente equivalentes en la población colombiana o en aquellos pacientes que viven en alturas por encima de $2000 \mathrm{msnm}$, en las cuales la presión arterial de oxígeno normal puede ser significativamente más baja $(69 \mathrm{~mm} \mathrm{Hg})$. El presente estudio evalúa la concordancia entre las tres escalas mencionadas, en dichas condiciones.

\section{MÉTODOS}

La población de estudio fueron los pacientes entre 18 y 65 años con diagnóstico de pancreatitis aguda, quienes recibieron atención en el Hospital Universitario San Ignacio de Bogotá (Colombia), en el período comprendido entre 2012 y 2016. Asimismo, se excluyeron los pacientes con antecedentes de pancreatitis crónica y que tuvieran seguimiento intrahospitalario por menos de $48 \mathrm{~h}$, dado que en ellos no era posible calcular todas las escalas de riesgo. El protocolo fue aprobado por el comité de ética del Hospital Universitario San Ignacio y la Pontificia Universidad Javeriana.

Los datos de seguimiento de estos pacientes se recolectaron de forma sistemática, a partir de la información consignada en el sistema de historia clínica electrónica institucional. Se incluyeron variables demográficas, clínicas, examen físico y parámetros bioquímicos. El procesamiento de exámenes en el laboratorio clínico institucional ha mantenido las mismas técnicas de medición durante todo el tiempo del estudio. El cálculo de las escalas de riesgo se basó directamente en los reportes de laboratorio y en los datos de las características clínicas reportadas para cada momento del tiempo, y no en los cálculos consignados en la historia clínica por los médicos tratantes.

En cada paciente se calcularon tres escalas y se consideraron las manifestaciones presentes en el momento de su ingreso. Las escalas fueron APACHE II (2), Ranson (3) y Marshall (4). Sobre la base de esos resultados, los pacientes se estadificaron en pancreatitis con o sin predicción de gravedad. En ese sentido, se definió la pancreatitis con predicción de gravedad como un puntaje en la escala de APACHE II $\geq 8,3$ o más criterios de Ranson positivos o un puntaje de Marshall modificado $\geq 2$.

Además, se recolectó la información referente a desenlaces como mortalidad, lesión renal aguda, falla respiratoria, presencia de necrosis pancreática, colecciones líquidas agudas peripancreáticas, pseudoquistes pancreáticos, necesidad de intervención quirúrgica y los días de estancia hospitalaria.

Las variables continuas se expresan como media y desviación estándar $(\mathrm{DE})$ si siguen una distribución normal, y como mediana y rango intercuartílico (RIC) si no lo hacen. Entre tanto, las variables categóricas se describen como porcentajes. Para analizar la concordancia entre los diferentes métodos de clasificación se utilizó el índice kappa. La interpretación de este coeficiente como evaluador de la fuerza de la concordancia es la siguiente: 0,01-0,20 (pobre), 0,210,40 (débil), 0,41-0,60 (moderada), 0,61-0,80 (buena), 0,81-0,90 (muy buena) y 0,9-1,00 (casi perfecta) (5). Se fijó un nivel de significación alfa de 0,05. Los cálculos se realizaron con ayuda del programa estadístico Stata $15^{\circ}$.

\section{RESULTADOS}

Se incluyeron 200 pacientes, cuyas características demográficas se describen en la Tabla 1. El 20,5\% de ellos presentaron complicaciones durante su hospitalización, bien sea necrosis, colecciones líquidas agudas peripancreáticas, pseudoquistes pancreáticos o necesidad de intervención quirúrgica. Tres pacientes fallecieron durante el seguimiento.

El porcentaje de los pacientes que fueron clasificados en pancreatitis con predicción de gravedad, para cada escala 
Tabla 1. Características demográficas de los pacientes incluidos

\begin{tabular}{|c|c|}
\hline Variable & $n=200$ \\
\hline Edad (años), media (DE) & $54,4(19)$ \\
\hline Sexo femenino, $n(\%)$ & $113(56,2)$ \\
\hline Lesión renal, n (\%) & $39(19,4)$ \\
\hline $\begin{array}{l}\text { Días de estancia, } n(\%) \\
\quad<5 \text { días } \\
6-10 \text { días } \\
11-15 \text { días } \\
>16 \text { días }\end{array}$ & $\begin{array}{l}51(25,5) \\
95(47,5) \\
20(10) \\
34(17)\end{array}$ \\
\hline $\begin{array}{l}\text { Presión arterial media }(\mathrm{mm} \mathrm{Hg}), \mathrm{n} \\
\quad<65 \\
65-90 \\
>90\end{array}$ & $\begin{array}{c}6(3) \\
85(42,5) \\
109(54,5)\end{array}$ \\
\hline $\begin{array}{l}\text { PaFi, } n(\%) \\
\quad<100 \\
100-300 \\
300-500 \\
\text { Sin datos }\end{array}$ & $\begin{array}{c}85(44,04) \\
107(55,4) \\
1(0,52) \\
7(3,5)\end{array}$ \\
\hline $\begin{array}{l}\text { Creatinina }(\mathrm{mg} / \mathrm{dL}), \mathrm{n}(\%) \\
\quad<1,5 \\
\quad 1,5-3 \\
>3\end{array}$ & $\begin{array}{c}178(89) \\
19(9,5) \\
1(0,5)\end{array}$ \\
\hline $\begin{array}{l}\text { Hematocrito al ingreso (\%), n (\%) } \\
\quad<30 \\
30-40 \\
40-50 \\
>50\end{array}$ & $\begin{array}{c}3(1,5) \\
30(15) \\
124(62) \\
43(21,5)\end{array}$ \\
\hline $\begin{array}{l}\text { Hematocrito a las } 48 \mathrm{~h}(\%), \mathrm{n}(\%) \\
\quad<30 \\
30-40 \\
40-50 \\
>50 \\
\quad \text { Sin datos }\end{array}$ & $\begin{array}{c}4(2) \\
87(43,5) \\
82(41) \\
7(3,5) \\
20(10)\end{array}$ \\
\hline $\begin{array}{l}\text { GOT }(\mathrm{U} / \mathrm{L}), \mathrm{n}(\%) \\
\quad<200 \\
>200 \\
\text { Sin datos }\end{array}$ & $\begin{array}{c}128(64) \\
66(33) \\
6(1)\end{array}$ \\
\hline $\begin{array}{l}\text { GPT }(\mathrm{U} / \mathrm{L}), \mathrm{n}(\%) \\
\quad<200 \\
>200 \\
\quad \text { Sin datos }\end{array}$ & $\begin{array}{c}137(68,5) \\
54(27) \\
9(4,5)\end{array}$ \\
\hline $\begin{array}{l}\text { Secuestro de líquidos (L), n (\%) } \\
\quad<4 \\
4-10 \\
>10 \\
\text { Sin datos }\end{array}$ & $\begin{array}{l}87(43,5) \\
80(40) \\
30(15) \\
3(1,5)\end{array}$ \\
\hline Presencia de comorbilidad, n (\%) & $41(20,5)$ \\
\hline Muerte, $\mathrm{n}(\%)$ & $3(1,5)$ \\
\hline
\end{tabular}

DE: desviación estándar; $\mathrm{n}$ : número; $\mathrm{mm} \mathrm{Hg}$ : milímetros de mercurio; PaFi: relación de presión arterial de oxígeno sobre fracción inspirada de oxígeno; mmol/L: milimoles por litro; U/L: unidades por litro; GOT: transaminasa glutámico-oxalacética (Glutamic Oxaloacetic Transaminase); GPT: transaminasa glutámico pirúvica (Glutamic Pyruvic Transaminase) de riesgo, se presenta en la Tabla 2. La escala de Marshall fue la que clasificó más pacientes en pancreatitis con predicción de gravedad (45,5\%), mientras que el 13,5\% de los pacientes tuvieron un puntaje APACHE II $>12$.

Tabla 2. Categorías según las escalas APACHE II, Ranson y Marshall

\begin{tabular}{|cc|}
\hline \multicolumn{1}{|c|}{ Variable } & $\mathbf{n = 2 0 0}$ \\
\hline APACHE II, $n(\%)$ & \\
Sin PDG & $121(60,5)$ \\
Con PDG & $79(39,5)$ \\
\hline Ranson, $n(\%)$ & \\
Sin PDG & $123(61,5)$ \\
Con PDG & $77(38,5)$ \\
Marshall, $n(\%)$ & \\
Sin PDG & $109(54,5)$ \\
Con PDG & $91(45,5)$ \\
\hline
\end{tabular}

APACHE II: Acute Physiology and Chronic Health disease Classification System II; PDG: predicción de graveyard

De otro lado, el coeficiente kappa mostró un acuerdo débil (kappa: 0,201; IC, 95 \%: 0,05-0,34). Los pacientes concordantes con pancreatitis con predicción de gravedad fueron $40(20 \%)$, mientras que $76(38 \%)$ fueron discordantes. De estos últimos, $37(18,5 \%)$ se clasificaron en pancreatitis con predicción de gravedad a partir de la escala de Ranson, pero no por la APACHE II, y 39 (19,5\%) fueron categorizados en pancreatitis con predicción de gravedad mediante la escala APACHE II y no por Ranson. A continuación, se describe la evaluación de la concordancia entre las escalas de APACHE y Ranson (Tabla 3).

Por su parte, el acuerdo entre APACHE II y Marshall fue moderado, con un coeficiente de kappa de 0,42 (IC, $95 \%$ : 0,28-0,56). Los pacientes concordantes clasificados en pancreatitis con predicción grave fueron $57(28,5 \%)$. De los 56 pacientes discordantes $(26,5 \%)$, la mayoría pertenecía al grupo categorizado en pancreatitis con predicción grave de la escala Marshally no de APACHE II (34 pacientes, 15,5\%) (Tabla 4).

Asimismo, la evaluación de concordancia entre la escala de Ranson y la de Marshall demostró un acuerdo pobre, con un coeficiente kappa de 0,18 (IC, 95 \%: 0,04-0,32). Los pacientes concordantes clasificados en pancreatitis con predicción de gravedad fueron 44 ( $22 \%$ del total), mientras que los 80 casos discordantes (40\%) correspondieron a 47 casos categorizados en pancreatitis con predicción de gravedad por Marshall y no por Ranson (23,5 \%), y 33 pacientes fueron clasificados en pancreatitis con predicción de gravedad mediante la escala de Ranson y no por Marshall (16,5 \%) (Tabla 5). 
Tabla 3. Concordancia entre las escalas APACHE II y Ranson

\begin{tabular}{|c|c|c|c|c|}
\hline & & \multicolumn{2}{|c|}{ Ranson } & \multirow[t]{2}{*}{ Total } \\
\hline & & Pancreatitis sin PDG & Pancreatitis con PDG & \\
\hline \multirow{3}{*}{ APACHE ॥ } & $\begin{array}{l}\text { Pancreatitis sin PDG } \\
\mathrm{n}(\% \text { del total) }\end{array}$ & $84(42)$ & $37(18,5)$ & $121(60,5)$ \\
\hline & $\begin{array}{l}\text { Pancreatitis con PDG } \\
\mathrm{n}(\% \text { del total) }\end{array}$ & $39(19,5)$ & $40(20)$ & $79(39,5)$ \\
\hline & $\begin{array}{l}\text { Total } \\
\mathrm{n}(\% \text { del total })\end{array}$ & $123(61,5)$ & $77(38,5)$ & $200(100)$ \\
\hline
\end{tabular}

Kappa: 0,201; IC, 95 \%: 0,05-0,34. APACHE II: Acute Physiology and Chronic Health disease Classification System II; PDG: predicción de gravedad

Tabla 4. Concordancia entre las escalas APACHE II y Marshall

\begin{tabular}{|c|c|c|c|c|}
\hline & & \multicolumn{2}{|c|}{ Marshall } & \multirow[t]{2}{*}{ Total } \\
\hline & & Pancreatitis sin PDG & Pancreatitis con PDG & \\
\hline \multirow{3}{*}{ APACHE II } & $\begin{array}{l}\text { Pancreatitis sin PDG } \\
\mathrm{n}(\% \text { del total })\end{array}$ & $87(43,5)$ & $34(15,5)$ & $121(60,5)$ \\
\hline & $\begin{array}{l}\text { Pancreatitis con PDG } \\
\mathrm{n}(\% \text { del total })\end{array}$ & $22(11)$ & $57(28,5)$ & $79(39,5)$ \\
\hline & $\begin{array}{l}\text { Total } \\
\mathrm{n}(\% \text { del total })\end{array}$ & $109(54,5)$ & $91(45,5)$ & $200(100)$ \\
\hline
\end{tabular}

Kappa: 0,42; IC, 95 \%: 0,28-0,56. APACHE II: Acute Physiology and Chronic Health disease Classification System II; PDG: predicción de gravedad

Tabla 5. Concordancia entre las escalas Ranson y Marshall

\begin{tabular}{|c|c|c|c|c|}
\hline & & \multicolumn{2}{|c|}{ Marshall } & \multirow[t]{2}{*}{ Total } \\
\hline & & Pancreatitis sin PDG & Pancreatitis con PDG & \\
\hline \multirow{3}{*}{ Ranson } & $\begin{array}{l}\text { Pancreatitis sin PDG } \\
\mathrm{n}(\% \text { del total })\end{array}$ & $76(38)$ & $47(23,5)$ & $123(61,5)$ \\
\hline & $\begin{array}{l}\text { Pancreatitis con PDG } \\
\mathrm{n}(\% \text { del total) }\end{array}$ & $33(16,5)$ & $44(22)$ & $77(38,5)$ \\
\hline & $\begin{array}{l}\text { Total } \\
\mathrm{n}(\% \text { del total })\end{array}$ & $109(54,5)$ & $91(45,5)$ & $200(100)$ \\
\hline
\end{tabular}

Kappa: 0,18; IC, 95 \%: 0,04-0,32. PDG: predicción de gravedad

\section{DISCUSIÓN}

Este es el primer estudio en Colombia -y en pacientes que viven en altitudes por encima de $2000 \mathrm{msnm}$-, que compara los resultados obtenidos al clasificar la severidad de la pancreatitis aguda, con las tres escalas de riesgo más usadas, con lo cual se demuestra que la concordancia es baja. Por tanto, estos resultados no deberían interpretarse como clínicamente equivalentes.
Nuestra población de estudio evidenció que la mayoría de los pacientes fueron mujeres entre los 46 y 65 años. La mortalidad global durante nuestro estudio fue del $1,5 \%$, cifra que es levemente inferior a lo publicado en la literatura $(2-3 \%)(6)$. Esto puede asociarse a que nosotros decidimos incluir solo pacientes menores de 65 años.

$\mathrm{Al}$ evaluar individualmente cada escala se observa que, según la de Marshall, el 45,5\% de los pacientes corresponderían a pancreatitis con predicción de gravedad, mientras 
que de acuerdo con la de APACHE II el 39,5\% de presentarían esa misma condición, y en la de Ranson serían el 38,5 \% de ellos. Es llamativo que, en nuestro estudio, el porcentaje de pacientes con pancreatitis severa es mayor al reportado en múltiples estudios anteriores, en los cuales, en promedio, se encontró una proporción de pacientes entre el 8 y $15 \%$ (7).

Dicho hallazgo puede estar asociado a que nuestra institución es de referencia, y a que decidimos incluir solo pacientes que hubiesen tenido un seguimiento de por lo menos $48 \mathrm{~h}$, lo que pudo generar que los pacientes con pancreatitis leve quedaran pobremente representados. Para la escala de Marshall, esta alta proporción de pacientes clasificados como severos puede también relacionarse con que nuestra institución se ubica a $2670 \mathrm{msnm}$, lo cual genera que, con frecuencia, los pacientes sean categorizados como severos, sobre la base de la puntuación que dan las bajas presiones de oxígeno arterial.

Todo ello sugiere que debería buscarse un punto de corte diferente para la presión arterial de oxígeno en ciudades por encima de los $2000 \mathrm{msnm}$, y que la escala de Marshall debería ser validada en estas condiciones antes de utilizarse.

La diferencia en la proporción de pacientes clasificados en pancreatitis con predicción de gravedad, evidenciada en este estudio, ya nos muestra que muchos enfermos que se incluyeron en esta categoría, mediante la escala de Marshall, no recibirían el mismo diagnóstico en las otras escalas. Esto hace que no sea posible para el médico tratante definir cuál de las clasificaciones es más acorde con el riesgo del paciente y, por tanto, establecer cuál sería el manejo recomendable.

Entre tanto, en nuestro estudio se documentó una pobre concordancia entre la escala de Ranson y las de APACHE II y Marshall, mientras que la concordancia fue levemente mejor entre APACHE y Marshall. En general, se puede concluir que existe una baja concordancia entre las escalas referidas, por lo que no pueden interpretarse como clínicamente equivalentes en los pacientes con pancreatitis.

Así, este hallazgo conduce a implicaciones clínicas importantes en la toma de decisiones durante el servicio de urgencias. Las directrices prácticas sugieren que, para definir la enfermedad como pancreatitis con predicción de gravedad, es necesario un valor $\geq 8$ puntos en la escala de APACHE II, así como 3 o más criterios de Ranson, o 2 o más en la escala de Marshall modificada.

Sin embargo, si aceptamos estos parámetros podríamos enfrentarnos a dos escenarios clínicos. El primero de ellos es aquel en el cual el médico decide realizar procedimientos diagnósticos y terapéuticos, como el traslado y el manejo en cuidado intensivo, a los pacientes que se clasifiquen en pancreatitis con predicción de gravedad, según cualquiera de las escalas. Esta estrategia, que se caracteriza por tener una alta sensibilidad, pero una baja especificidad, generaría un gasto inútil para el sistema de salud, dado que se realizarían intervenciones innecesarias en algunos pacientes.

El segundo caso clínico es aquel en el cual, por el contrario, se decide actuar a partir del resultado de una de las escalas, pero se ignoran las otras dos. Si esto se realizara así, una cantidad significativa de pacientes, que se beneficiarían de intervenciones adicionales, podrían quedarse sin manejo. Dicha situación podría causar un aumento de la mortalidad y de las complicaciones.

Estos hallazgos ya eran esperables, si evaluamos el rendimiento diagnóstico reportado por otros autores sobre las diferentes escalas. Así, para la escala de Ranson se registró una sensibilidad del $63 \%$, una especificidad del $76 \%$, un valor predictivo positivo del $79 \%$ y un valor predictivo negativo del $92 \%$. Mientras tanto, para la escala APACHE II se han documentado una sensibilidad del $82 \%$, una especificidad del $86 \%$, un valor predictivo positivo del $81 \%$ y un valor predictivo negativo del $98 \%$ (8).

En el ámbito latinoamericano, existen estudios que han arrojado resultados que contradicen los datos reportados en el presente trabajo. En Perú, Ponce (9) realizó un estudio observacional con 77 pacientes, para evaluar la concordancia de tres escalas de pronóstico de severidad (APACHE II, Ranson y BISAP). Esta investigación encontró una concordancia casi perfecta entre las escalas, con porcentajes que coincidían por encima del $90 \%$, y fue especialmente buena entre la de BISAP y APACHE II. Así pues, en dicho estudio se recomienda usar cualquiera de las escalas anteriores, pero siempre considerando que aquella más fácil de aplicar para el médico tratante debe ser la primera opción.

En 2005, Rosas y colaboradores realizaron otro estudio similar en México (10). En él se evaluaron las escalas APACHE II, Osborne, Ranson, otras pruebas paraclínicas de severidad (hematocrito, calcio sérico y déficit de base) y los hallazgos tomográficos de Balthazar. El estudio descrito concluyó que tanto la sensibilidad como la especificidad de cada escala son similares a las que han sido reportadas en la literatura mundial. Sin embargo, este trabajo también enfatiza en la necesidad de continuar desarrollando estudios como el nuestro, así como de validación externa, en vista de que ninguna investigación ha evaluado las tres escalas no solo en la población colombiana, sino latinoamericana en general.

Por otra parte, los resultados divergentes pueden estar asociados a que existen diferencias en los sistemas de salud. De igual manera, hay diferencias en el momento del tiempo en que se realizan los estudios o en las características biológicas de una población y otra. Por tanto, sería necesario validar las distintas escalas en cada nación y, probablemente también, en diversos contextos de un mismo país (11).

Nuestro estudio tiene limitaciones asociadas al tamaño de la muestra, que es relativamente pequeña, y al bajo 
número de eventos de mortalidad o de complicaciones. Por tanto, no es posible realizar una validación de las escalas en Colombia. Serán necesarios estudios con un tamaño de muestra significativamente más grande, a fin de lograr este objetivo.

Una segunda consecuencia de este tamaño de muestra limitado es que los IC son relativamente amplios. Sin embargo, las conclusiones obtenidas sobre la base del análisis de la concordancia serían similares en ambos extremos de los intervalos; es decir que no se puede asumir que las interpretaciones de los resultados de las escalas sean clínicamente equivalentes.

Además, nosotros no incluimos pacientes mayores de 65 años, por lo que nuestros resultados no pueden aplicarse a esta población. Se requieren nuevos estudios para evaluar si la concordancia entre las diferentes escalas cambia de forma significativa.

Una limitación adicional del estudio está dada por el sesgo de selección, consecuencia de que en nuestra población quedó subrepresentado el grupo de pacientes con pancreatitis sin predicción de gravedad. Esto sugiere que nuestros datos podrían no aplicar a instituciones con menor nivel de complejidad.

Asimismo, nuestros resultados demuestran que la concordancia entre las escalas de APACHE II, Ranson y Marshall es baja y que, por tanto, no deberían interpretarse como clínicamente equivalentes en Colombia. También sugieren que se debe plantear un punto de corte menor en los niveles de presión arterial de oxígeno, cuando se desea usar la escala de Marshall en ciudades por encima de 2000 msnm, a fin de evitar clasificar de forma equivocada como severos a una proporción alta de pacientes.

Dado que no existen estudios de validación que nos permitan definir cuál de ellas es más apropiada para ser usada en nuestro medio, se hace énfasis en que, aparte de la puntuación en la escala predictiva de severidad escogida, el médico tratante debe siempre evaluar los factores de riesgo independientes de cada paciente e individualizar el caso, para lo cual requiere utilizar paraclínicos e imágenes adicionales.

Desde nuestro punto de vista, es preferible la estrategia de clasificar como pancreatitis con predicción de gravedad un caso de pancreatitis, si resulta positivo para cualquiera de las escalas descritas. La prioridad debe ser la seguridad de los pacientes antes que los costos de atención.

\section{Conflictos de interés}

Los autores declaran no tener conflictos de interés.

\section{Fuente de financiación}

Los autores.

\section{REFERENCIAS}

1. Huerta-Mercado J. Tratamiento médico de la pancreatitis aguda. Rev Med Hered. 2013;24(3):231-236. https://doi.org/10.20453/rmh.v24i3.320

2. Larvin M, McMahon MJ. APACHE-II score for assessment and monitoring of acute pancreatitis. Lancet. 1989;2(8656):201-205. http://doi.org/10.1016/s0140-6736(89)90381-4

3. Ranson JH, Spencer FC. Prevention, diagnosis, and treatment of pancreatic abscess. Surgery. 1977;82(1):99-106.

4. Banks PA, Bollen TL, Dervenis C, Gooszen HG, Johnson CD, Sarr MG, Tsiotos GG, Vege SS; Acute Pancreatitis Classification Working Group. Classification of acute pancreatitis--2012: revision of the Atlanta classification and definitions by international consensus. Gut. 2013;62(1):102-11.

http://doi.org/10.1136/gutjnl-2012-302779

5. Landis JR, Koch GG. The measurement of observer agreement for categorical data. Biometrics. 1977;33(1):159-174. http://doi.org/10.2307/2529310

6. Werner J, Feuerbach S, Uhl W, Büchler MW. Management of acute pancreatitis: from surgery to interventional inten- sive care. Gut. 2005;54(3):426-436.

http://doi.org/10.1136/gut.2003.035907

7. Everhart J. Pancreatitis. In: The burden of digestive diseases in the United States. US Department of Health and Human Services. 2004. p. 119-122.

8. Wu BU, Johannes RS, Sun X, Tabak Y, Conwell DL, Banks PA. The early prediction of mortality in acute pancreatitis: a large population-based study. Gut. 2008;57(12):1698-1703. http://doi.org/10.1136/gut.2008.152702

9. Ponce-Monar CR. Concordancia que existe entre tres escalas de predictores de severidad en pancreatitis aguda en pacientes atendidos en el servicio de medicina interna en el Hospital María Auxiliadora en el periodo 2016. Lima: Universidad Privada San Juan Bautista; 2017.

10. Rosas FM, Gaxiola WR, Ibáñez GO, Vargas TE, Meza VM, Calvo IJ. Evaluación de las escalas y factores pronóstico en pancreatitis aguda grave. Cir Gen. 2005;27(2):137-143.

11. Altman DG, Vergouwe Y, Royston P, Moons KG. Prognosis and prognostic research: validating a prognostic model. BMJ. 2009;338:b605. http://doi.org/10.1136/bmj.b605 